\title{
Are Orthotrichum and Dorcadion (Orthotrichaceae, Bryophyta) heterotypic generic names?
}

\author{
Vítězslav Plášek, Jakub Sawicki \& Ryszard Ochyra
}

\begin{abstract}
Are Orthotrichum and Dorcadion (Orthotrichaceae, Bryophyta) heterotypic generic names? - Acta Mus. Siles. Sci. Natur. 65: 193-202, 2016.

Abstract: The generic name Dorcadion Adans. ex Lindb. is a superfluous and illegitimate name for Orthotrichum Hedw. The new generic name Dorcadionella Plášek, Sawicki \& Ochyra is proposed for a segregate of Orthotrichum which comprises species with superficial stomata that are always monoicous and only occasionally produce gemmae on leaves. Dorcadionella affinis (Schrad. ex Brid.) Plášek, Sawicki \& Ochyra is selected as a type of this new generic name. The genus Dorcadionella consists of 63 species and one variety that have a worldwide distribution. Traditionally, they were all placed in Orthotrichum and the relevant transfers to the new genus are effected in the present account.
\end{abstract}

Key words: Adanson; bryophytes; Dorcadionella gen. nov.; illegitimate name; Musci; new combinations; nomenclature; moss taxonomy

\section{Introduction}

Orthotrichum Hedw. is one of the largest of all moss genera. Since its inception (Hedwig 1801) over 250 species have been given names under this generic name, of which about 150 species are still accepted in modern bryology (Lewinsky 1993; Crosby et al. 2000). Because species of this genus exhibit great diversity of morphological traits, Orthotrichum was subdivided into no fewer than seven subgenera and five sections (Vitt 1971; Lewinsky 1993). However, according to recent molecular studies only five subgenera are now recognised (Sawicki et al. 2012).

Despite remarkable diversity of morphologies, in classical taxonomy the genus was given cohesion by a specific combination of primarily sporophyte characters. These include immersed to short-exserted and often ribbed capsules with a diplolepidous peristome consisting of 16 or 8 narrow endostome segments alternating with 16 or 8 paired exostome teeth, which have thickened, usually ornamented outer plates and mostly thinner and less ornamented inner plates. The characteristic shape of capsules and the peristome structure is coupled with large, campanulate-mitrate and mostly hairy calyptrae which entirely cover the capsules and this unique combination of morphological traits makes Orthotrichum one of the most distinctive and readily recognisable of all moss genera. In addition, the plants have orthotropic, mostly sympodially branched stems and they exhibit typically xerophytic features, including small, isodiametric and mostly papillose cells with incrassate walls. This is associated with the ecological predilections of Orthotrichum species which generally grow in open and sunny habitats, either epiphytically on bark of deciduous trees or epipetrically on rock faces and stones. As a result, any of the species described since the last decade of the eighteenth century have been accurately placed in Orthotrichum and hence this generic name almost entirely lacks synonyms.

\section{Taxonomic history of the generic name Orthotrichum}

Because Orthotrichum species are distinguished on the basis of microscopic characters, they were recognised relatively late. Dillenius (1741) in his Historia muscorum described only three species for which he used polynomial names and which are currently recognised as 
O. affine Schrad. ex Brid., O. stramineum Brid. or O. rupestre Schwägr. (Lindberg 1883). The first of these is the earliest known species of the genus which had been described for the first time by Morison (1699) as Muscus humilis tectorum subfuscus, capitulis brevibus, pileolis erectis, pediculis curtis, the second was described for the first time by Petiver (1703) as Adianthum aureum acaulon, pileis strictis, whilst the third was discovered by Dillenius (1741) in the vicinity of Bangor, Wales, and recognised as Polytrichum capsulis sessilibus, foliis brevibus, rectis, carinatis.

Linnaeus (1753) accepted only one species distinguished by Dillenius (1741) and named it Bryum striatum L. and the other two considered as its unnamed varieties. In contrast, Adanson (1763) accepted three Dillenian species and placed them in the separate genus Dorcadion Adans. but did not use the binomial names for them.

Hedwig (1789) established the new genus Orthotrichum and placed three species in it, two of which are still treated in that genus: $O$. striatum Hedw. and $O$. anomalum Hedw. In fact, it is generally overlooked that the first description of Orthotrichum was provided by Timm (1788), who dedicated his Florae Megapolitanae prodromus to J. Hedwig, and it is rather evident that he had received from him the relevant information on Orthotrichum which had been then scheduled for publication in the second volume of Descriptio et adumbratio microscopico-analytica muscorum frondosorum. This volume was published only a year later (Hedwig 1789) and Timm (1788) gave references to it in the genus and species names. These pre-1801 names were subsequently validated by Hedwig (1801) in his Species muscorum frondosorum, the nomenclatural starting-point work for mosses (Sphagnum excepted). Finally, Grout (1935: 106) designated O. anomalum as the generitype of Orthotrichum.

The characteristic combination of morphological traits has made Orthotrichum one of the most distinctive and readily recognisable of all moss genera and new species accurately placed in it. The upshot of this ostensible homogeneity of this genus was that its name has only a few synonyms, including Nauia Borkh., Brachytrichum Röhl., Dorcadion Lindb., Muelleriella Dusén, nom. cons., Stroemia I. Hagen, hom. illeg. (三 Nyholmiella Holmen \& E.Warncke) and Orthomitrium Lewinsky-Haapasaari \& Crosby. Of these, Muelleriella and Nyholmiella are generally accepted as distinct genera (Vitt 1976; Sawicki et al. 2010; Plášek et al. 2015).

Recent phylogenetic studies have revealed the polyphyletic nature of Orthotrichum and provided strong evidence for the division of species of this genus into groups characterised by immersed versus superficial stomata (Goffinet et al. 1998, 2004; Sawicki et al. 2009, 2010). In particular, phylogenomic analysis based on complete mitochondrial genomes and platomes, as well as nuclear rRNA gene clusters, have fully supported splitting the traditionally conceived genus Orthotrichum into two additional segregates (Sawicki et al. 2016 /in press/).

As a result, the monotypic genus Pulvigera Plášek, Sawicki \& Ochyra was recognised to accommodate $P$. lyellii (Hook. \& Taylor) Plášek, Sawicki \& Ochyra as a sole species and for species with phaneropore (superficial) stomata, monoicous sex condition, mostly lacking gemmae and having a haploid chromosome number of 6, the generic name Dorcadion Adans. ex Lindb. was adopted. The molecular data also suggest that species with superficial stomata are more closely allied with species of Ulota Mohr than with species of Orthotrichum sensu strictu with cryptopore (immersed) stomata and having a haploid chromosome number of 11 (with one known exception) (cf. Plášek et al. 2015).

The application of the generic name Dorcadion for this large segregate of Orthotrichum (Plášek et al. 2015) proved to be incorrect. This name has had a chequered nomenclatural history and Lindberg's (1878) discussion on the genus Dorcadion, which was written in the old-fashioned Swedish, was initially misinterpreted. Closer re-examination of this text revealed that the intent of Lindberg (1878) was different and he clearly showed the identity of Dorcadion and Orthotrichum. 


\section{An historical account of the generic name Dorcadion}

The genus name Dorcadion was introduced by Adanson (1763) as one of eight new names he coined for genera of mosses. In fact, he ascribed this name to Dioscorides and it is

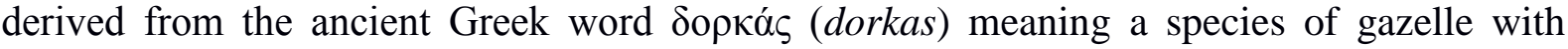
distinctive dark and white bands running along the sides of the animal. In orthotrichalean mosses this name apparently makes a reference to distinct longitudinal ridges and colourful bands on the capsule typical of many species. As is the case with other Adanson (1763) names, Dorcadion had fallen into obsolescence for over a century. It was apparently because the orthotrichalean mosses were then still inadequately known and the genus itself was poorly characterised by Adanson (1763). In the meantime Hedwig (1789) described in detail the new genus Orthotrichum which gained universal acceptance after using it in Species muscorum frondosorum (Hedwig 1801).

The generic name Dorcadion was resurrected by Lindberg (1878) who provided the following justification for his decision:

"Af de nya äro alla sammansatta af ganska heterogena länkar och derföronaturliga, med undantag af två, Sekra och Dorcadion, det förra alldeles detsamma som Cinclidotus P.-B., det sednare fullkomligt liktydigt med Orthotrichum Hedw. (our bold), Brid., fastän de lemnade diagnoserna visserligen icke äro så välgenomförda som vetenskapen numera kräfver. Vi anse oss likväl nödgade at åter upptaga dess tvänne genusnamn, dels emedan de äro ganska naturligt begrämsade såsom omslutande endast sins emellan nära affina arter, dels för prioritetsrättens konsequenta genomförande, dels äfven på grund af att de flesta af hans fröväxtsjägten ånyo kommit till heder, blot med en obetydlig förändring i stafningssättet, der välljudet sådant fordrade, hvarpä Genera plantarum af Bentham och Hooker lemna talrika bevis, såsom t. ex. Asimina, Canavalia, Foeniculum, Arctostaphylos, Carbenia, Carduncellus, Encelia, Liabum, Oftia m. fl., samt slutligen emedan åtskilliga af hans nya genera bland fanerogamerna säkerligen icke äro bättre skilldrade än sagda två inom mossornas grupp, men det oaktadt blifvit gillade".

The translation from Swedish reads: "All of the new ones [genera proposed by Adanson] are connected by fairly heterogeneous links and are therefore unnatural, with the exception of two, Sekra and Dorcadion, the first mentioned quite the same as Cinclidotus P.-B., the latter exactly the same as Orthotrichum Hedw. (our bold), Brid., although the diagnoses are not as accurate as nowadays demanded by science. We still feel forced to reinstate these two genus names, first since they are rather naturally delimited and include only closely related species, second because of the consequent application of the priority rule, and third since most of his [Adanson's] seed plant genera have again come into use, with small changes in spelling, when the pronunciation demanded this, as shown in numerous cases in Genera plantarum by Bentham and Hooker, e.g. Asimina, Canavalia, Foeniculum, Arctostaphylos, Carbenia, Carduncellus, Encelia, Liabum, Oftia, and so on, and finally since several of his new genera among the phanerogams are certainly not better described than the mentioned two among the bryophytes, and are still accepted".

Apart from the above discussion, Lindberg (1878) provided the following diagnosis of Dorcadion which was compiled from the data presented by Adanson (1863) in table form: "Dorcadion. Feuilles alternes triangulaires. Fleurs mâles solitaires, axillaires sessiles. Fleurs femelle ..... Anteres ovoïde avec un opercule et une coëfe velue. Graines ..... - Polytrichon Dill. Hist. musc., tab. 55, figg. 8-10". In modern taxonomy these species are recognised as Orthotrichum affine, $O$. stramineum and $O$. rupestre, respectively.

The words "det sednare fullkomligt liktydigt med Orthotrichum Hedw.", which mean "the latter exactly the same as Orthotrichum Hedw.", make Dorcadion Adans. ex Lindb., quite unambiguously, a superfluous and illegitimate name for Orthotrichum Hedw. according to the modern rules of nomenclature (McNeill et al. 2012). However, in the light of the then 
mandatory rules of nomenclature the statement on the identity of Dorcadion and Orthotrichum meant that the former name should have been adopted for orthotrichalean mosses. Accordingly, Lindberg (1879) transferred all Scandinavian species of Orthotrichum to Dorcadion.

Lindberg's $(1878,1879)$ nomenclatural novelties were largely ignored by the vast majority of contemporary bryologists and the name Orthotrichum unanimously continued to be used in all major floras and compendia of mosses (e.g. Lesquereux \& James 1884; Venturi 1887-1888; Braithwaite 1889; Limpricht 1890; Brown 1895; Paris 1895, 1905; Dixon \& Jameson 1896; Brotherus 1902; Roth 1904). The unlawful use of Orthotrichum was sanctioned in 1910 when the Third International Botanical Congress in Brussels changed the starting-point for nomenclature of mosses (Sphagnum excepted) to 1801, the year of the publication of Hedwig's Species muscorum frondosorum and this decision remains mandatory (McNeill et al. 2012). Accordingly, Orthotrichum was legally reinstated as a proper genus name for this large group of mosses and Dorcadion must be considered as a homotypic synonym (Wijk et al. 1962; Farr et al. 1979).

The answer to the question in the title of this article is obviously an unequivocal "No". With Lindberg's (1878) reference to Orthotrichum Hedw., it is not the final sentence of Art. 10.2 that is relevant, but Art. 52.2(c) and Art. 7.5 of the current (Melbourne) Code (McNeill et al. 2012). By stating that Dorcadion was the same as Orthotrichum, Lindberg (1878) was also making indirect reference (Art. 10.3) to O. crispum Hedw., O. anomalum Hedw., and $O$. striatum Hedw. Accordingly, without conservation with a different type, which is hardly a serious option, Dorcadion is not available for use.

Orthotrichum Hedw., Sp. Musc. Frond.: 162. 1801. Type: Orthotrichum anomalum Hedw. (vide Grout 1935: 106).

$\equiv$ Dorcadion Adans. ex Lindb., Utkast Eur. Bladmoss.: 7. 1878, nom. illeg. incl. gen. prior. $\equiv$ “Orthotrichum Hedw. [unranked] Dorcadion Kindb.”, Bih. Kongl. Svenska Vetensk.-Akad. Handl. 7(9): 118, 122. 1883, nom. inval. (Art. 22.2).

\section{Nomenclatural implications}

The illegitimacy of the generic name Dorcadion implies that for a segregate of the traditionally understood Orthotrichum, consisting of exclusively monoicous species with phaneroporous stomata, no name is available. Accordingly, Dorcadionella is proposed as a new generic name to accommodate the species which were incorrectly positioned under Dorcadion by Plášek et al. (2015). This new name is a diminutive of Dorcadion.

Dorcadionella Plášek, Sawicki \& Ochyra, gen. nov.

Genus hoc generi Orthotricho simile sed stomatibus phaneroporis et plantis semper monoicis gemmas rarissime efferentibus facillime dignoscendum. Type: Dorcadionella affinis (Schrad. ex Brid.) Plášek, Sawicki \& Ochyra, comb. nov. BASIONYM: Orthotrichum affine Brid., Muscol. Recent. 2(2): 22. 1801.

Species of Dorcadionella are characterised by having appressed to erect leaves that are narrowly lanceolate to oblong- or ovate-lanceolate, keeled, acuminate to acute or rounded with the margins mostly recurved. The costa is single and usually subpercurrent. Short cylindrical, unbranched gemmae are occasionally present on the leaves. Sex condition is always monoicous. The capsules are immersed to somewhat long exserted, smooth or less often furrowed when dry. The stomata are superficial. The vaginula is naked or hairy. The peristome is double (rarely reduced or single), erect or reflexed when dry. It consists of 16 exostome teeth that are usually arranged in 8 pairs and an endostome of 8 or 16 segments. 
The calyptra is naked or sparsely to densely hairy. A haploid chromosome number of 6 has been determined in species which have hitherto been studied chromosomally.

Dorcadionella is a medium size genus which currently consists of 63 species and one variety which have a worldwide distribution. Originally, they were all described under the generic name Orthotrichum and herein they are given names in Dorcadionella. This necessitates the following new names and combinations. Only names currently used are listed.

Dorcadionella acuminata (H.Philib.) Plášek, Sawicki \& Ochyra, comb. nov. Basionym: Orthotrichum acuminatum H.Philib., Rev. Bryol. 8: 28. 1881.

Dorcadionella affinis (Schrad. ex Brid.) Plášek, Sawicki \& Ochyra var. bohemica (Plášek \& Sawicki) Plášek, Sawicki \& Ochyra, comb. nov.

Basionym: Orthotrichum affine Schrad. ex Brid. var. bohemicum Plášek \& Sawicki, Čas. Slezsk. Zemsk. Muzea, Vědy Př́r., Série A, 61(1): 1. 2012.

Dorcadionella anaglyptodon (Cardot \& Broth.) Plášek, Sawicki \& Ochyra, comb. nov. Basionym: Orthotrichum anaglyptodon Cardot \& Broth., Bih. Kongl. Svenska Vetensk.-Akad. Handl. 63(10): 31, t. 2 f. 10. 1923.

Dorcadionella araucarieti (Müll.Hal. ex Broth.) Plášek, Sawicki \& Ochyra, comb. nov. Basionym: Orthotrichum araucarieti Müll.Hal. ex Broth., Ergebn. Bot. Exped. Südbras., Musci: 287. 1924.

Dorcadionella arborescens (Thér. \& Naveau) Plášek, Sawicki \& Ochyra, comb. nov. Basionym: Orthotrichum arborescens Thér. \& Naveau, Bull. Soc. Roy. Bot. Belgique 60: 48. 1927.

Dorcadionella armata (Lewinsky \& van Rooy) Plášek, Sawicki \& Ochyra, comb. nov. Basionym: Orthotrichum armatum Lewinsky \& van Rooy, J. Bryol. 16: 69, f. 2-5. 1990.

Dorcadionella bolanderi (Sull.) Plášek, Sawicki \& Ochyra, comb. nov. Basionym: Orthotrichum bolanderi Sull., Icon. Musc. Suppl.: 64, pl. 46. 1874.

Dorcadionella brassii (E.B.Bartram) Plášek, Sawicki \& Ochyra, comb. nov. Basionym: Orthotrichum brassii E.B.Bartram, Lloydia 5: 268, f. 25. 1942.

Dorcadionella brotheri (Dusén ex Lewinsky) Plášek, Sawicki \& Ochyra, comb. nov. Basionym: Orthotrichum brotheri Dusén ex Lewinsky, Mem. New York Bot. Gard. 45: 348, f. 133-143. 1987.

Dorcadionella cyathiformis (R.Br.bis) Plášek, Sawicki \& Ochyra, comb. nov. Basionym: Orthotrichum cyathiforme R.Br.bis, Trans. Proc. New Zealand Inst. 27: 436, t. 39f. 24. 1895.

Dorcadionella dasymitrium (Lewinsky) Plášek, Sawicki \& Ochyra, comb. nov. Basionym: Orthotrichum dasymitrium Lewinsky, Bryobrothera 1: 169, f. 1-2. 1992.

Dorcadionella densa (Lewinsky) Plášek, Sawicki \& Ochyra, comb. nov. Basionym: Orthotrichum densum Lewinsky, Mem. New York Bot. Gard. 45: 336, f. 51-62. 1987.

Dorcadionella elegans (Schwägr. ex Hook. \& Grev.) Plášek, Sawicki \& Ochyra, comb. nov. Basionym: Orthotrichum elegans Schwägr. ex Hook. \& Grev., Edinburgh J. Sci. 1: 122, pl. 6 [upper right]. 1824.

Dorcadionella elegantula (Schimp. ex Mitt.) Plášek, Sawicki \& Ochyra, comb. nov. Basionym: Orthotrichum elegantulum Schimp. ex Mitt., J. Linn. Soc., Bot. 12: 187. 1869.

Dorcadionella elongata (Taylor) Plášek, Sawicki \& Ochyra, comb. nov. Basionym: Orthotrichum elongatum Taylor, London J. Bot. 5: 45. 1846.

Dorcadionella erosa (Lewinsky) Plášek, Sawicki \& Ochyra, comb. nov. Basionym: Orthotrichum erosum Lewinsky, J. Hattori Bot. Lab. 72: 32, f. 17. 1992. 
Dorcadionella fenestrata (Cardot \& Thér.) Plášek, Sawicki \& Ochyra, comb. nov.

Basionym: Orthotrichum fenestratum Cardot \& Thér., Proc. Washington Acad. Sci. 4(5): 310-311, pl. 16 f. 2. 1902.

Dorcadionella firma (Venturi) Plášek, Sawicki \& Ochyra, comb. nov. Basionym: Orthotrichum firmum Venturi, Nuovo Giorn. Bot. Ital. 4: 15. 1872.

Dorcadionella galiciae (F.Lara, Garilleti \& Mazimpaka) Plášek, Sawicki \& Ochyra, comb. nov.

Basionym: Orthotrichum galiciae F.Lara, Garilleti \& Mazimpaka, Nova Hedwigia 83: 200, f. 1-11. 2006.

Dorcadionella graphiomitrium (Müll.Hal. ex Beckett) Plášek, Sawicki \& Ochyra, comb. nov. Basionym: Orthotrichum graphiomitrium Müll. Hal. ex Beckett, Trans. Proc. New Zealand Inst. 25: 291, f. 36. 1893.

Dorcadionella hawaiica (Müll.Hal.) Plášek, Sawicki \& Ochyra, comb. nov. Basionym: Orthotrichum hawaiicum Müll.Hal., Flora 82: 451. 1896.

Dorcadionella holzingeri (Renauld \& Cardot) Plášek, Sawicki \& Ochyra, comb. nov. Basionym: Orthotrichum holzingeri Renauld \& Cardot, Contr. U.S. Natl. Herb. 3(4): 270. 1895.

Dorcadionella hookeri (Wilson ex Mitt.) Plášek, Sawicki \& Ochyra, comb. nov. Basionym: Orthotrichum hookeri Wilson ex Mitt., J. Proc. Linn. Soc., Bot., Suppl. 1: 48. 1859.

Dorcadionella hortensis (Bosw.) Plášek, Sawicki \& Ochyra, comb. nov. Basionym: Orthotrichum hortense Bosw., J. Bot. 30: 97. 1892.

Dorcadionella iberica (F.Lara \& Mazimpaka) Plášek, Sawicki \& Ochyra, comb. nov. Basionym: Orthotrichum ibericum F.Lara \& Mazimpaka, Nova Hedwigia 56: 263, f. 1-3. 1993.

Dorcadionella incana (Müll.Hal.) Plášek, Sawicki \& Ochyra, comb. nov. Basionym: Orthotrichum incanum Müll.Hal., Flora 68: 419. 1885.

Dorcadionella incurvomarginata (Lewinsky \& van Rooy) Plášek, Sawicki \& Ochyra, comb. nov.

Basionym: Orthotrichum incurvomarginatum Lewinsky \& van Rooy, J. Bryol. 16: 67, f. 1, 4. 1990.

Dorcadionella iwatsukii (Ignatov) Plášek, Sawicki \& Ochyra, comb. nov. Basionym: Orthotrichum iwatsukii Ignatov, Arctoa 10: 172. 2001.

Dorcadionella johnstonii (E.B.Bartram) Plášek, Sawicki \& Ochyra, comb. nov. Basionym: Orthotrichum johnstonii E.B. Bartram, Trav. Bryol. 1[13]: 132. 1942.

Dorcadionella keeverae (H.A.Crum \& L.E.Anderson) Plášek, Sawicki \& Ochyra, comb. nov. Basionym: Orthotrichum keeverae H.A. Crum \& L.E. Anderson, J. Elisha Mitchell Sci. Soc. 72(2): 281-284, f. 3-12. 1956.

Dorcadionella laevigata (J.E.Zetterst.) Plášek, Sawicki \& Ochyra, comb. nov. Basionym: Orthotrichum laevigatum J.E.Zetterst., Öfvers. Kongl. Vetensk.-Akad. Förh. 19(5): 363. 1862.

Dorcadionella latimarginata (Lewinsky) Plášek, Sawicki \& Ochyra, comb. nov. Basionym: Orthotrichum latimarginatum Lewinsky, Mem. New York Bot. Gard. 45: 331, f. 14-24. 1987.

Dorcadionella laxifolia (Wilson in Mitt.) Plášek, Sawicki \& Ochyra, comb. nov. Basionym: Orthotrichum laxifolium Wilson in Mitt., Hooker's J. Bot. Kew Gard. Misc. 3: 57. 1851.

Dorcadionella leiolecythis (Müll.Hal.) Plášek, Sawicki \& Ochyra, comb. nov. Basionym: Orthotrichum leiolecythis Müll.Hal., Nuovo Giorn. Bot. Ital., N. Ser. 3: 107. 1896. 
Dorcadionella ludificans (Lewinsky) Plášek, Sawicki \& Ochyra, comb. nov. Basionym: Orthotrichum ludificans Lewinsky, Mem. New York Bot. Gard. 45: 347, f. 122-132. 1987.

Dorcadionella mandonii (Schimp. ex Hampe) Plášek, Sawicki \& Ochyra, comb. nov. Basionym: Orthotrichum mandonii Schimp. ex Hampe, Ann. Sci. Nat. Bot., Sér. 5, 4: 332. 1865.

Dorcadionella oreophila (Lewinsky \& van Rooy) Plášek, Sawicki \& Ochyra, comb. nov. Basionym: Orthotrichum oreophilum Lewinsky \& van Rooy, J. Bryol. 16: 74, f. 4, 6. 1990.

Dorcadionella pariata (Mitt) Plášek, Sawicki \& Ochyra, comb. nov. Basionym: Orthotrichum pariatum Mitt., J. Linn. Soc., Bot. 12: 186. 1869.

Dorcadionella praemorsa (Venturi) Plášek, Sawicki \& Ochyra, comb. nov. Basionym: Orthotrichum praemorsum Venturi, Bot. Centralbl. 44(13): 418. 1890.

Dorcadionella psychrophila (Mont.) Plášek, Sawicki \& Ochyra, comb. nov. Basionym: Orthotrichum psychrophilum Mont., Ann. Sci. Nat., Bot., Sér. 2, 9: 52. 1838.

Dorcadionella pulchra (Lewinsky) Plášek, Sawicki \& Ochyra, comb. nov. Basionym: Orthotrichum pulchrum Lewinsky, J. Hattori Bot. Lab. 72: 20, f. 1-10. 1992.

Dorcadionella pycnophylla (Schimp. ex Müll.Hal.) Plášek, Sawicki \& Ochyra, comb. nov. Basionym: Orthotrichum pycnophyllum Schimp. ex Müll.Hal., Syn. Musc. Frond. 1: 709. 1849.

Dorcadionella pylaisii (Brid.) Plášek, Sawicki \& Ochyra, comb. nov. Basionym: Orthotrichum pylaisii Brid., Bryol. Univ. 1: 722.1826 ['Pylaisaei'].

Dorcadionella rupestris (Schwägr.) Plášek, Sawicki \& Ochyra, comb. nov. Basionym: Orthotrichum rupestre Schwägr., Sp. Musc. Frond., Suppl. 1(2): 27, pl. 53 [top]. 1816.

Dorcadionella sainsburyi (Allison) Plášek, Sawicki \& Ochyra, comb. nov. Basionym: Orthotrichum sainsburyi Allison, Trans. Proc. Roy. Soc. New Zealand 77: 280. 1947.

Dorcadionella schoddei (Lewinsky) Plášek, Sawicki \& Ochyra, comb. nov. Basionym: Orthotrichum schoddei Lewinsky, J. Hattori Bot. Lab. 72: 34, f. 20.1992.

Dorcadionella shawii (Wilson in Schimp.) Plášek, Sawicki \& Ochyra, comb. nov. Basionym: Orthotrichum shawii Wilson in Schimp., Musci Eur. Nov. Fasc. 1-2: 17, t. 652 [Mon.: 1, t. 1]. Dorcadionella sordida (Sull. \& Lesq.) Plášek, Sawicki \& Ochyra, comb. nov. Basionym: Orthotrichum sordidum Sull. \& Lesq. in Austin, Musci Appalach.: 30. 1870.

Dorcadionella spanotricha (Lewinsky) Plášek, Sawicki \& Ochyra, comb. nov. Basionym: Orthotrichum spanotrichum Lewinsky, Lindbergia 18: 116, f. 1-2. 1994.

Dorcadionella speciosa (Nees) Plášek, Sawicki \& Ochyra, comb. nov. Basionym: Orthotrichum speciosum Nees in Sturm, Deutschl. Fl., Abth. II, Cryptog. 5(17): pl. 5. 1819.

Dorcadionella spjutii (D.H.Norris \& Vitt) Plášek, Sawicki \& Ochyra, comb. nov. Basionym: Orthotrichum spjutii D.H.Norris \& Vitt, Nova Hedwigia 56: 260, f. 1. 1993.

Dorcadionella striata (Hedw.) Sawicki \& Ochyra, comb. nov. Basionym: Orthotrichum striatum Hedw., Sp. Musc. Frond.: 163. 1801.

Dorcadionella subulata (Mitt.) Plášek, Sawicki \& Ochyra, comb. nov. Basionym: Orthotrichum subulatum Mitt., J. Linn. Soc., Bot. 12: 184. 1869.

Dorcadionella taiwanensis (Lewinsky) Plášek, Sawicki \& Ochyra, comb. nov. Basionym: Orthotrichum taiwanense Lewinsky, J. Hattori Bot. Lab. 72: 25, f. 12. 1992. 
Dorcadionella tasmanica (Hook.f. \& Wilson) Plášek, Sawicki \& Ochyra, comb. nov. Basionym: Orthotrichum tasmanicum Hook.f. \& Wilson, London J. Bot. 7: 27, pl. 1c. 1848.

Dorcadionella tenuicaulis (Lewinsky) Plášek, Sawicki \& Ochyra, comb. nov. Basionym: Orthotrichum tenuicaule Lewinsky, J. Hattori Bot. Lab. 75: 45, f. 1. 1994.

Dorcadionella tortidontia (F.Lara, Garilleti \& Mazimpaka) Plášek, Sawicki \& Ochyra, comb. nov.

Basionym: Orthotrichum tortidontium F.Lara, Garilleti \& Mazimpaka, Nova Hedwigia 63: 517, f. 1-17. 1996.

Dorcadionella tortifolia (Lewinsky) Plášek, Sawicki \& Ochyra, comb. nov. Basionym: Orthotrichum tortifolium Lewinsky, J. Hattori Bot. Lab. 72: 23, f. 11. 1992.

Dorcadionella truncata (Lewinsky \& Deguchi) Plášek, Sawicki \& Ochyra, comb. nov. Basionym: Orthotrichum truncatum Lewinsky \& Deguchi, Bryologist 89: 216, f. 1-17. 1987.

Dorcadionella truncatodentata (Müll.Hal.) Plášek, Sawicki \& Ochyra, comb. nov. Basionym: Orthotrichum truncatodentatum Müll.Hal., Linnaea 43: 442. 1882 ['truncato-dentatum'].

Dorcadionella undulata Plášek, Sawicki \& Ochyra, nom. nov.

Breplaced synonym: Orthotrichum undulatum Mitt., hom. illeg., J. Linn. Soc. Bot. 12: 187. 1869.

Dorcadionella vicaria (Laz.) Plášek, Sawicki \& Ochyra, comb. nov. Basionym: Orthotrichum vicarium Laz., Žurn. Inst. Bot. Vseukrajins'k. Akad. Nauk 26-27: 210. 1938.

Dorcadionella vladikavkana (Venturi) Plášek, Sawicki \& Ochyra, comb. nov. Basionym: Orthotrichum vladikavkanum Venturi in Husnot, Muscol. Gall.: 167. 1887.

Acknowledgements: We are very grateful to Dr John McNeill, Edinburgh, United Kingdom, for his valuable discussion of the nomenclatural issues and to Rod Seppelt, Bundall, Queensland, Australia for linguistic corrections of the manuscript. Thanks are also due to Lars Hedenäs, Stockholm, and Johannes Enroth, Helsinki, for translating the Swedish text. The contribution by V. Plášek is part of a research project of the Institute of Environmental Technologies, reg. no. CZ.1.05/2.1.00/03.0100; Project LO1208 of the National Feasibility Programme I of the Czech Republic. The contribution of R. Ochyra gained financial support through the statutory fund of the W. Szafer Institute of Botany of the Polish Academy of Sciences.

\section{References}

Adanson M. (1763): Familles des plantes. Vol. 2. Vincent, Paris, 640 pp.

Braithwaite R. (1889): Orthotrichum Hedw., 65-88 pp., lv-lviii Tab. In The British moss-flora. Part XII. Fam. X. Grimmiaceae. II. Published by the author, London.

Brotherus V.F. (1902): Orthotrichum Hedw., 466-472 pp. In Engler A. \& Prantl K. (eds), Die natürlichen Pflanzenfamilien nebst ihren Gattungen und wichtigeren Arten inbesondere den Nutzpflanzen. I. Teil. 3. I. Hälfte: Musci (Laubmoose). III. Unterklasse Bryales. I. Gruppe: Acrocarpi. Wilhelm Engelmann, Leipzig.

Brown R. (1895): Notes on the New Zealand mosses: genus Orthotrichum. - Transactions of the New Zealand Institute 27: 422-446 + pls. xxxv-xlii.

Crosby M.R., Magill R.E., Allen B. \& He S. (2000): A checklist of the mosses. Missouri Botanical Garden, St. Louis, $320 \mathrm{pp}$.

Dillenius J.J. (1741): Historia muscorum in qua circiter sexcentae species veteres novae ad sua genera relatae describuntur et iconibus genuinis illustrantur cum appendice et indice synonymorum. E Theatro Sheldoniano, Oxonii, xvi + 576 pp., lxxxv pls.

Dixon H.N. \& Jameson H.G. (1896): The student's handbook of British mosses. V.T. Sumfield, Eastbourne and John Wheldon \& Co., London, xlvi + 520 pp., i-xl pls.

Farr E.R., Leussink J.A. \& Stafleu F.A. (1979): Index nominum genericorum (plantarum). Volume 1 (Aa Epochnium). Regnum Vegetabile 100. Bohn, Scheltema \& Holkema, Utrecht and dr. W. Junk b.v., Publishers, The Hague, xxvi +630 pp.

Goffinet B., Bayer R.J. \& Vitt D.H. (1998): Circumscription and phylogeny of the Orthotrichales (Bryopsida) inferred from $r b c L$ sequence analyses. - American Journal of Botany 85: 1324-1337. 
Goffinet B., Shaw A.J., Cox C.J., Wickett N.J. \& Boles S. (2004): Phylogenetic inferences in the Orthotrichoideae (Orthotrichaceae: Bryophyta) based on variation in four loci from all genomes. Monographs in Systematic Botany from the Missouri Botanical Garden 98: 270-289.

Grout A.J. (1935): Family Orthotrichaceae, 106-145 pp., pls. 45-62. In Grout, A.J. (ed.), Moss flora of North America north of Mexico. Vol. 2, parts 2-3. Privately published, Newfane, Vermont.

Hedwig J. (1789): Descriptio et adumbratio microscopico-analytica muscorum frondosorum nec non aliorum vegetatntium e classe cryptogamica Linnaei novorum dubiisque vexatorum. Vol. 2. - In bibliopolio I. G. Mülleriano, Lipsiae, i + 112 pp., Tab. i-xl.

- (1801): Species muscorum frondosorum descriptae et tabulis aeneis LXXVII coloratis illustratae. Sumtu Joannis Ambrosii Barthii, Lipsiae and apud Amand Koenig, Parisiis, vi + 353 pp., lxxii pls.

Lesquereux L. \& James Th.P. (1884): Manual of the mosses of North America. S.E. Cassino and Company, Boston, v + 447 pp., i-vi pls.

Lewinsky J. (1993): A synopsis of the genus Orthotrichum Hedw. (Musci, Orthotrichaceae). - Bryobrothera 2: $1-59$.

Limpricht K.G. (1890): 81. Gattung: Orthotrichum Hedw. 34-102 pp. In Dr L. Rabenhorst's KryptogamenFlora von Deutschland, Oesterreich und der Schweiz. 2 Auflage. Vierter Band: Die Laubmoose Deutschlands, Oesterreichs und der Schweiz. II. Abtheilung: Bryineae (Stegocarpae [Acrocarpae, Pleurocarpae excl. Hypnaceae]). Eduard Kummer, Leipzig.

Lindberg S.O. (1878): Utkast till en naturlig gruppering af Europas bladmossor med toppsittande frukt (Bryinae acrocarpae). J. C. Frenckell \& Sons tryckeri, Helsingfors, 39 pp.

- (1879): Musci scandinavici in systemate novo naturali dispositi. Ex Officina Iesaiae Edquist, Upsaliae, 50 pp.

- (1883): Kritisk granskning af mossorna uti Dillenii Historia muscorum. J.C. Frenckell \& Son, Helsingfors, 59 pp.

Linnaeus C. (1753): Species plantarum, exhibentes plantas rite cognitas, ad genera relatas, cum differentiis specificis, nominibus trivialibus, synonymis selectis, locis natalibus, secundum systema sexuale digestas. Tom. 2. Impensis Laurentii Salvii, Holmiae, 561-1200 + [1-30 unnumbered, index $]$ pp.

McNeill J., Barrie F.R., Buck W.R., Demoulin V., Greuter W., Hawksworth D.L., Herendeen P.S., Knapp S., Marhold K., Prado J., Prud'homme van Reine W.F., Smith G.F., Wiersema J.H. \& Turland N.J. (eds) (2012): International Code of Nomenclature for algae, fungi, and plants (Melbourne Code), adopted by the Eighteenth International Botanical Congress Melbourne, Australia, July 2011. Regnum Vegetabile 154. Koeltz Scientific Books, Königstein, xxx +208 pp.

Morison R. (1699): Plantarum historiae universalis oxoniensis pars secunda [tertia] seu herbarum distributio nova, per tabulas cognationis \& affinitatis ex libro naturae observata \& detecta. E Theathro Sheldoniano, Oxonii, xxiv + 657 pp.

Paris E.G. (1895): Index bryologicus sive enumeratio muscorum hucusque cognitorum adjunctis synonyma distributioneque geographica locupletissimis. Pars 2. Libraire scientifique A. Hermann, Parisiis, 325-644 pp.

- (1905): Index bryologicus sive enumeratio muscorum ad diem ultimam anni 1900 cognitorum adjunctis synonyma distributioneque geographica locupletissimis. Ed. 2. Vol. 3. Parisiis: Paul Klinksieck, 400 pp.

Petiver J. (1703): Musei Petiveriani centuria X, rariora naturae continens, viz. animalia, fossilia, plantas, ex variis mundi plagis advecta, ordine digesta, et nominibus priopris signata. Typ. Smith and Walford, Londini, 93 pp., 2 pls.

Plášek V., Sawicki J., Ochyra R., Szczecińska M. \& Kulik T. (2015): New taxonomical arrangement of the traditionally conceived genera Orthotrichum and Ulota (Orthotrichaceae, Bryophyta). - Acta Musei Silesiae. Scientiae Naturales 64: 169-174.

Roth G. (1904): Die europäischen Laubmoose. Band 1. Kleistokarpische und Akrokarpische Moose bis zu den Bryaceen. Verlag von Wilhelm Engelmann, Leipzig, xiii + 598 pp., pls, i-lii.

Sawicki J., Plášek V. \& Szczecińska M. (2009): Preliminary studies on the phylogeny of the genus Orthotrichum inferred from nuclear ITS sequences. - Annales Botanici Fennici 46: 507-515.

- (2010): Molecular studies resolve Nyholmiella (Orthotrichaceae) as a separate genus. - Journal of Systematics and Evolution 48: 183-194.

- (2012): Molecular data do not support the current division of Orthotrichum (Bryophyta) species with immersed stomata. - Journal of Systematics and Evolution 50: 12-24.

Sawicki J., Plášek V., Ochyra R., Szczecińska M., Myszczyński K. \& Kulik T. (2016): Mitogenomic analyses support the recent division of the genus Orthotrichum (Orthotrichaceae, Bryophy-ta). - International Journal of Molecular Sciences 17: in press.

Timm J.Ch. (1788): Florae Megapolitanae prodromus exhibens plantas ducatus megapolitano-suerinensis spontaneas. Maxime secundum systema Linneano-Thunbergianum digestas. Apud heredes I. G. Mulleri, Lipsiae, xvi + 284 [+ 285-298, index]. 
Venturi [A.] (1887-1888): LIII. Orthotrichum Hedw., 154-196 pp. + pls. xliii-lii. In Husnot T., Muscologia gallica. Descriptions et figures des mousses de France et de contrées voisines. Prèmiere partie - Acrocarpes. T. Husnot, Cahan and F. Savy, Paris.

Vitt D.H. (1971): The infrageneric evolution, phylogeny, and taxonomy of the genus Orthotrichum (Musci) in North America. - Nova Hedwigia 21: 683-711.

- (1976): A monograph of the genus Muelleriella Dusén. - The Journal of the Hattori Botanical Laboratory 40: 91-113.

Wijk R. van der, Margadant W.D. \& Florschütz P.A. (1962): Index muscorum. Volume 2 (D-Hypno). Regnum Vegetabile 26. International Bureau for Plant Taxonomy and Nomenclature, Utrecht, $535 \mathrm{pp}$.

Authors' addresses: Vítězslav Plášek, Dept. of Biology and Ecology, University of Ostrava, Chittussiho 10, CZ-710 00 Ostrava, Czech Republic.

E-mail: vitezslav.plasek@osu.cz

Jakub Sawicki, Department of Botany and Nature Protection, University Warmia and Mazury, Plac Łódzki 1, 10-728 Olsztyn, Poland and Dept. of Biology and Ecology, University of Ostrava, Chittussiho 10, CZ-710 00 Ostrava, Czech Republic.

E-mail: jakub.sawicki@uwm.edu.pl

Ryszard Ochyra, Laboratory of Bryology, Institute of Botany, Polish Academy of Sciences, Ul. Lubicz 46, 31-512 Kraków, Poland.

E-mail: r.ochyra@botany.pl 